\title{
Accuracy of parents' subjective assessment of paediatric fever with thermometer measured fever in a primary care setting
}

\author{
George Edwards ${ }^{1 *}$, Susannah Fleming ${ }^{1}$, Jan Y. Verbakel ${ }^{2}$, Ann van den Bruel ${ }^{2}$ and Gail Hayward ${ }^{1}$
}

\begin{abstract}
Background: Fever is a common symptom of benign childhood illness but a high fever may be a sign of a serious infection. Temperature is often used by parents to check for illness in their children, and the presence of a high temperature can act as a prompt to consult a healthcare professional. It would be helpful for GPs to understand how well parental assessment of the presence of fever correlates with temperature measurement in the clinic in order to incorporate the history of the child's fever into their clinical assessment.
\end{abstract}

Methods: Secondary analysis of a cross-sectional diagnostic method comparison study. Parents were asked whether they thought their child had fever before their temperature was measured by a researcher. Fever was defined as a temperature of $38^{\circ} \mathrm{C}$ and higher using either an axillary or tympanic thermometer.

Results: Of 399 children recruited, 119 (29.8\%) were believed by their parents to be febrile at the time of questioning and 23 (6.3\%) had a fever as measured by a researcher in the clinic. 23.5\% of children with a parental assessment of fever were found to have a fever in the clinic. Less than $1 \%$ of children whose parents thought they did not have a fever were found to be febrile in the clinic. Having more than one child did not improve accuracy of parents assessing fever in their child.

Conclusions: In the GP surgery setting, a child identified as afebrile by their parent is highly likely to be measured as such in the clinic. A child identified as febrile by their parent is less likely to be measured as febrile.

\section{Introduction}

Fever is a common symptom in benign childhood illnesses [1]. It is often used by parents to confirm illness in a child [2]. Fevers are a source of concern and worry for parents [3]. As a sign of more severe illness, which in rare cases may lead to brain damage and death $[4,5]$, fever in a child acts as a prompt for a GP visit [6-8]. From a GP perspective, parents presenting with febrile children are common. In out-of-hours care almost a third of contacts for children under the age of 12 are fever related $[9,10]$

\footnotetext{
*Correspondence: george.edwards@phc.ox.ac.uk

${ }^{1}$ Nuffield Department of Primary Care Health Sciences, Radcliffe

Observatory Quarter, Woodstock Road, Oxford OX2 6GG, UK
}

Full list of author information is available at the end of the article whilst fever accounts for $20 \%$ of all visits to the paediatric emergency department [11]. Current clinical guidelines also use fever thresholds as part of algorithms for admission decisions [7] meaning temperature levels affect GPs' management decisions. Single assessment of a child's temperature in a GP surgery may not be the most informative way to measure fever, particularly given the poor agreement between different thermometer types [12] and prior administration of antipyretics by parents.

Parents of children presenting to primary care are likely to have assessed their child's fever [13] and it is useful for GPs to understand the previous duration and trajectory of a child's fever. It would be helpful to know how well parental assessment of the presence of fever correlates with temperature measurement in the clinic in order to 
incorporate the history of the fever into a holistic assessment of the child.

This study is a secondary analysis of a larger methods comparison study which found wide limits of agreement between infrared, axillary and tympanic thermometry [12]. In the method comparison study we concluded that peripheral temperature measurement should be interpreted cautiously and within the context of a holistic assessment of the child. In this secondary analysis, we aim to establish whether parental assessment of fever correlated with temperature measurement in a primary care setting.

\section{Methods}

We used data collected during a prospective cross-sectional diagnostic method comparison study comparing two non-contact infrared thermometers with electronic axillary and tympanic thermometers [12]. Children between 0 and 5 years with any acute illness of a maximum of 14 days presenting to general practice ( 9 sites) or an out-of-hours $(\mathrm{OOH})$ general practice service (1 site) in Oxfordshire (UK) were eligible for inclusion. Demographic information (age, gender, ethnicity, number of siblings) and history of the illness (e.g. duration) were recorded by a research assistant before the child's temperature was measured using four thermometers. Parents were asked whether they thought their child had "fever now" (yes/no). If they answered in the affirmative, they were asked whether the child was "warm to touch", whether they had "measured temperature at home", or "other". Parents were also asked whether their child had received fever medication in the previous $6 \mathrm{~h}$, and if so what medication had been administered.

The sample size for the main study was calculated at 533 to give a $0.75^{\circ} \mathrm{C}$ accuracy for each limit of agreement. This was reduced to 400 to give a $0.10^{\circ} \mathrm{C}$ accuracy.

We calculated sensitivity, specificity, and positive and negative predictive values using a $2 \times 2$ table against a threshold of $38^{\circ} \mathrm{C}$ using either an electronic axillary or tympanic thermometer. Since there is little evidence of agreement between axillary and tympanic thermometry [12], and both have similar agreement with rectal temperatures $[14,15]$, we used a combined reference standard for this study. We did a further sub-group analysis of the sensitivity, specificity, and positive and negative predictive values of parents with only one child and those with more than one child. We used R (3.5.1) for our analyses.

\section{Results \\ Descriptive}

We recruited 401 children between April 2017 and August 2018 and we obtained an axillary or a tympanic thermometer reading for 399 children with a median age of 19.8 months (IQR 9.5-40.6); 202 (50.6\%) were boys (see Table 1 for an age group distribution) and 234 (58.6\%) children had siblings. We were unable to obtain a temperature reading with either axillary or tympanic thermometers from two children. Using a threshold of $38.0^{\circ} \mathrm{C}$ with either the axillary or tympanic thermometer $30 / 399(7.5 \%)$ of children were febrile.

The parents of $119 / 399$ (29.8\%) children thought their child had a fever at the time of asking. Of these, 67/119 $(56.3 \% \%)$ indicated that their child felt 'warm to touch', $41 / 119$ (34.5\%) had measured the temperature of their child at home, 10/119 (8.4\%) used both techniques, and $1 / 119(0.8 \%)$ did not specify. The parents of $134 / 399$ (33.6\%) children gave an antipyretic in the 6 hours prior to professional assessment in the clinic. The parents of $75 / 134(56.0 \%)$ of these children receiving an antipyretic thought they had a fever.

\section{Methods comparison}

Against a reference standard of $38^{\circ} \mathrm{C}$ using either an axillary or tympanic thermometer, parents' subjective assessment of fever was associated a sensitivity of $93 \%$ (95\% CI $73-99 \%)$, a specificity of $75 \%$ (95\% CI 71-80\%), a positive predictive value of $24 \%$ (95\% CI $16-32 \%$ ) and a negative predictive value of $99 \%$ (95\% CI $97-100 \%$ ) The positive likelihood ratio was 3.78 (95\% CI 3.09-4.63), and the negative likelihood ratio was 0.09 (95\% CI 0.02-0.34). See Table 2 and Supplementary Table 1 for further details.

We analysed the subgroups of 165 parents with one child versus 234 with more than one child (Table 3, full data in Supplementary Table 2). The sensitivity, specificity, and NPV in each group was similar. The PPV of parents with one child was $31 \%$ (95\% CI 19-46) in comparison to $18 \%$ (95\% CI 10-29) for parents with more than one child.

\section{Discussion \\ Summary of results}

Of 399 children recruited to our study with axillary or tympanic thermometer measurements 119 (29.8\%) were believed to be febrile by their parents. Only 30 (7.5\%)

Table 1 Age distribution of 399 children with an axillary or tympanic temperature reading

\begin{tabular}{lll}
\hline Age group & $\boldsymbol{N}$ & $\%$ \\
\hline$<1$ month & 5 & $1.3 \%$ \\
$\geq 1$ month and $<3$ months & 20 & $5.0 \%$ \\
$\geq 3$ months and $<12$ months & 100 & $25.1 \%$ \\
Between 12 months and 5years & 274 & $68.7 \%$ \\
\hline
\end{tabular}


Table 2 Test characteristics of parents' subjective assessment of fever

\begin{tabular}{|c|c|c|c|c|c|c|}
\hline Index Test & Reference & $N=$ & TP & FP & $\mathrm{FN}$ & TN \\
\hline Parents' subjective assessment & $380 \mathrm{C}$ using either the axillary or tympanic thermometer & 399 & 28 & 91 & 2 & 278 \\
\hline
\end{tabular}

Table 3 Test characteristics of parents' subjective assessment of fever by family size

\begin{tabular}{|c|c|c|c|c|c|c|c|}
\hline Sub-Group & Index Test & Reference & $n=$ & TP & FP & FN & TN \\
\hline Parents with one child & Parental subjective assessment & $\begin{array}{l}380 C \text { using either the } \\
\text { axillary or tympanic } \\
\text { thermometer }\end{array}$ & 165 & 15 & 33 & 2 & 115 \\
\hline Parents with more than one child & Parental subjective assessment & $\begin{array}{l}380 C \text { using either the } \\
\text { axillary or tympanic } \\
\text { thermometer }\end{array}$ & 234 & 13 & 58 & 0 & 163 \\
\hline
\end{tabular}

children were found to have a temperature of $38^{\circ} \mathrm{C}$ or more measured using either the axillary thermometer or tympanic thermometer.

Compared with this reference standard, 93\% children found to have a fever in the clinic had been assessed as febrile by their parents (sensitivity). Of children assessed by their parents as having a fever, $24 \%$ were found to have a fever in the clinic (PPV) compared with only $1 \%$ of children whose parents through they were afebrile.

We examined whether parental experience, which we assumed to be greater in those with more than one child, would result in better diagnostic accuracy. The sensitivity, specificity, and NPV was similar in parents with one child and parents with more than one child. The PPV of parents with one child was $31 \%$ in comparison to $18 \%$ for parents with more than one child, although there were overlapping confidence intervals.

\section{Comparison with the literature}

A meta-analysis of tactile assessment of fever by caregivers found a combined sensitivity of $87.5 \%$, specificity of $54.6 \%$ [16], positive likelihood ratio of 1.93 (1.39-3.67), and a negative likelihood ratio of $0.23(0.15-0.36)$. Neither predictive value was calculated. A subsequent study conducted in a hospital found palpation to be associated with a sensitivity of $86 \%$, a specificity of $48 \%$, an NPV of $66 \%$ and a PPV of 76\% [17] although prevalence was not reported. Our results differ in the specificity and positive likelihood ratio, likely due to the setting and inclusion of children with non-febrile illness. Of the 11 studies included in the meta-analysis, four included patients specifically with fever as part of their complaint and none were conducted in primary care. In contrast we included all children presenting with acute illness, including those where fever might not be expected to be present by parents. This may have resulted in more parents correctly identifying a lack of fever. In support of this our study had more true negatives than the other studies.

One other study found parents' subjective assessment of fever in children presenting to ED to be associated with a sensitivity of $74 \%$, a specificity of $86 \%$, a PPV of $52 \%$ and an NPV of $94 \%$ [13]. The prevalence of fever in this study was higher than ours at $17 \%$. We found a similar sensitivity and specificity, but a lower PPV than in the $E D$, likely because we had a lower prevalence of fever.

\section{Clinical relevance}

Our results help clinician's incorporate a parents' assessment of fever into a holistic assessment of the child. A parent's subjective assessment of fever at presentation is a poor predictor of fever measured in primary care whilst a history of normal temperature in the current illness episode can however be seen as reasonably reliable by the GP.

\section{Limitations}

Our study has several limitations. In the UK, NICE Guidelines recommend measurement of temperature in children presenting with fever symptoms using electronic axillary thermometers, or tympanic thermometers in children aged 4 weeks and older [7]. Rectal measurements are no longer recommended yet both the axillary thermometer and the infrared tympanic thermometer are associated with wide limits of agreement with rectal temperature measurement $[14,15]$. Although comparison with axillary or tympanic thermometry is the most relevant comparison to current practice in the UK, use of these imperfect reference standards may limit international comparison.

Our study may also be underpowered to assess the usefulness of parent's subjective assessment of fever to GPs as it uses secondary data from a study with a different 
purpose [12] and we found a low number of children with a fever.

As we recruited before their initial assessment, we did not collect information on the underlying chief complain. Alongside the low prevalence found in our study for the presence of fever, this could limit the generalisability of our finds to high prevalence settings or to children with a different spectrum of presenting complaints.

\section{Further research}

Further research into how parents identify a fever in a home setting and how temperature readings are interpreted in the context of their child's illness will improve our understanding of actions performed by parents preceding contact with the health services. This understanding will enable clinicians to make better use of parents' assessment of their child's illness.

Further research is also needed into trajectories of body temperature over an illness episode in a child. This will help both parents and clinicians to contextualise and interpret a child's temperature in relation to the course of the illness.

\section{Conclusions}

In the GP surgery setting, a child identified as afebrile by their parent is highly likely to be measured as such in the clinic. A child identified as febrile by their parent is less likely to be measured as febrile.

\section{Supplementary Information}

The online version contains supplementary material available at https://doi. org/10.1186/s12875-022-01638-6.

Additional file 1: Supplementary Table 1. Full analysis. Supplementary Table 2. Full analysis by number of children.

\section{Acknowledgements}

GH was supported by an NIHR Academic Clinical Lectureship and GH and GE were supported by the NIHR Community Healthcare Medtech and IVD Cooperative. The Oxford Academic Health Science Network provided project management and funding support. The views expressed in this publication are those of the authors and not necessarily those of The Health Foundation, the NHS, the NIHR or the Department of Health and Social care.

\section{Authors' contributions}

GH and GE conceived of and designed the study, all authors contributed to the acquisition, analysis, and interpretation of the data. All authors have reviewed and approved the manuscript.

\section{Funding}

This project was funded by the HTA Programme (ref: 16/45/01). Ann van den Bruel, Gail Hayward, and George Edwards were supported by the National Institute for Health Research (NIHR) Community Healthcare MedTech and In Vitro Diagnostics Cooperative. The views expressed are those of the author(s) and not necessarily those of the NHS, the NIHR, or the Department of Health and Social Care. The sponsor had no role in study design, data collection, data analysis, data interpretation, writing of the report, or the decision to submit the paper for publication/.
Availability of data and materials

All data analysed as part of this study is available in the manuscript.

\section{Declarations}

\section{Ethics approval and consent to participate}

Ethical approval was given by South Central — Berkshire Research Ethics Committee (ref: 17/SC/0068). All methods were performed in accordance with the relevant guidelines and regulations. Informed consent for participation was obtained from the parents or legal guardian of all study subjects.

\section{Consent for publication}

Not applicable.

\section{Competing interests}

The authors declare that they have no competing interests.

\section{Author details}

${ }^{1}$ Nuffield Department of Primary Care Health Sciences, Radcliffe Observatory Quarter, Woodstock Road, Oxford OX2 6GG, UK. ${ }^{2}$ Academic Centre for Primary Care, Department of Public Health and Primary Care, KU Leuven, Leuven, Belgium.

Received: 10 May 2021 Accepted: 18 January 2022

Published online: 21 February 2022

\section{References}

1. Hay AD, Heron J, Ness A, Team TA study. The prevalence of symptoms and consultations in pre-school children in the Avon longitudinal study of parents and children (ALSPAC): a prospective cohort study. Fam Pract. 2005:22:367-74.

2. Ertmann RK, Reventlow S, Söderström M. Is my child sick? Parents' management of signs of illness and experiences of the medical encounter: parents of recurrently sick children urge for more cooperation. Scand J Prim Health Care. 2011;29(1):23-7.

3. Crocetti M, Moghbeli N, Serwint J. Fever phobia revisited: have parental misconceptions about fever changed in 20 years? Pediatrics. 2001;107(6):1241-6

4. Schmitt BD. Fever phobia. Am J Dis Child. 1980;134(2):176.

5. Kai J. What worries parents when their preschool children are acutely ill, and why: a qualitative study. Bmj. 2011;313(7063):983-6.

6. May M, Brousseau DC, Nelson DA, et al. Why parents seek Care for Acute IIIness in the clinic or the ED: the role of health literacy. Acad Pediatr. 2018;18(3):289-26.

7. NICE. Fever in under 5's: Assesment and initial management. Clin Guidel. 2017; (May 2013). Available from: https://pathways.nice.org.uk/pathways/ fever-in-under-5s/symptoms-and-signs-of-specific-illnesses-in-childrenwith-fever\#content=view-node\%3Anodes-kawasaki-disease.

8. Kelly M, Sahm LJ, Shiely F, et al. Parental knowledge, attitudes and beliefs on fever: a cross-sectional study in Ireland. BMJ Open. 2017;7(7):1-7.

9. de Bont EGPM, Peetoom KKB, Moser A, et al. Childhood fever: a qualitative study on GPs' experiences during out-of-hours care. Fam Pract. 2015;32(4):449-55.

10. De Bont EGPM, Lepot JMM, Hendrix DAS, et al. Workload and management of childhood fever at general practice out-of-hours care: an observational cohort study. BMJ Open. 2015;5(5):1-6.

11. Armon K, Stephenson T, Gabriel V, et al. Determining the common medical presenting problems to an accident and emergency department. Arch Dis Child. 2001:84:390-2.

12. Hayward GN, Verbakel JY, Abakar Ismail F, et al. Non-contact infrared versus axillary and tympanic thermometers in children attending primary care. Br J Gen Pract. 2020;70(693):e236-44.

13. Banco L, Veltri D. Ability of mothers to subjectively assess the presence of fever in their children. Am J Dis Child. 1984;138(10):976-8.

14. Craig JV, Lancaster GA, Taylor S, et al. Infrared ear thermometry compared with rectal thermometry in children: a systematic review. Lancet. 2002;360(9333):603-9. 
15. Craig JV, Lancaster GA, Williamson PR, Smyth RL. Temperature measured at the axilla compared with rectum in children and young people: systematic review. Bmj. 2000;320(7243):1174-8.

16. Li YW, Zhou LS, Li X. Accuracy of tactile assessment of fever in children by caregivers: a systematic review and meta-analysis. Indian Pediatr. 2017;54(3):215-21.

17. Rosenbloom E, Balis C, Jacobson D, et al. A cross-sectional study on subjective fever assessment in children by palpation: are fathers as reliable as mothers? Emerg Med Int. 2020;2020:1-5.

\section{Publisher's Note}

Springer Nature remains neutral with regard to jurisdictional claims in published maps and institutional affiliations.

- fast, convenient online submission

- thorough peer review by experienced researchers in your field

- rapid publication on acceptance

- support for research data, including large and complex data types

- gold Open Access which fosters wider collaboration and increased citations

- maximum visibility for your research: over $100 \mathrm{M}$ website views per year

At BMC, research is always in progress.

Learn more biomedcentral.com/submissions 